\title{
Impact of Covid-19 on Hematological Practice: Challenges and Opportunities
}

\author{
Rajat $\operatorname{Kumar}^{1}$ (1)
}

Published online: 12 October 2020

(C) Indian Society of Hematology and Blood Transfusion 2020

As on Sept 27, 2020, the total number of coronavirus disease 2019 (Covid-19) cases crossed 32 million, with India ranking second highest at almost 6 million and global deaths predicted to cross 1 million, as per WHO [1]. This pandemic has posed a major challenge to hematology patients who often need specialized management. As we face these problems, the crisis also offers opportunities for improving patient care, research and education.

\section{Challenges}

Patients with hematological malignancies are often neutropenic, immunocompromised, and receiving chemotherapy. Results from New York demonstrate that mortality was higher in patients with cancer, especially hematological malignancies, than in other patients [2]. Those with non-malignant disorders, like aplastic anemia or immune thrombocytopenia, are often also on immunosuppressive drugs and conceivably at higher risk. Due to the pandemic, severe disruptions have occurred to hematology services, compromising the care of patients with severe forms of thalassemia, sickle cells disease and hemophiliawho are dependent on regular blood component therapy and specialized supportive care. Patients too often delay seeking medical attention, presenting with advanced disease.

Rajat Kumar

rajat.kumar@uhn.ca

1 Department of Medical Oncology and Hematology, Princess Margaret Cancer Centre, UHN, University of Toronto, 610 University Avenue, 700U: 6-525, Toronto, ON M5G 2M9, Canada
Within weeks of the pandemic being declared, professional societies rallied and issued guidelines based on evolving evidence and expert opinion. These are aimed at reducing spread of the virus and managing with limited facilities as these are prioritized to treat Covid-19 patients. One of the best resources is provided by the American Society of Hematology (ASH), and it links to updated information from many other organizations at https://www. hematology.org/covid-19. While most of these measures work well in high-income countries, these may not be practical in economically deprived regions. The recent prioritized approach by Jain et al. [3] is commendable as this presents an Indian context and even includes Ministry of Health and Family Welfare recommendations. Their review will be valuable to other countries with similar infrastructure limitations.

There are other challenges faced by the hematology community worldwide. Voluntary blood donation has dropped due to fears of contracting the SARS-COv-2 in a health care facility. The blood component shortages provide an opportunity to use alternative measures like tranexamic acid to manage and prevent bleeding, erythropoiesis stimulating agents instead of red cell transfusions, and new triggers for platelet transfusions. The use of convalescent plasma from recovered patients of Covid-19 requires the services of transfusion medicine and apheresis teams with an impact on patients requiring apheresis facilities.

The ongoing pandemic will have consequences on the training of residents in hematology, who are now managing Covid-19 patients. Over the next year or more, this will affect a generation of clinical and laboratory physicians and scientists. Many clinical trials evaluating therapeutic discoveries for hematological disorders will suffer, as accrual to clinical trials has reduced while basic and 
translational research is hampered as resources are reprioritized.

\section{Opportunities}

Despite these challenges, there are also opportunities which should be explored. The adverse outcome of Covid-19 is due to an abnormal inflammatory response causing lung injury, respiratory distress and multi-organ failure. Another critical component is activation of the coagulation system. Elevated d-dimers and abnormal coagulation parameters are poor prognostic indicators. It is unclear if the coagulation abnormalities are a consequence of the inflammatory response or a direct and specific effect of the virus. These are areas of research where the laboratory and clinical hematologists can contribute. Anticoagulant agents have been proposed to reduce disease severity and we need data on the best anticoagulant, optimal duration of therapy, and possible bleeding complications. M Aggarwal et al. [4] have done a comprehensive review of hemostatic abnormalities in Covid-19, which will serve as an important resource to both clinicians and researchers.

Virtual clinics are a boon to patients travelling long distances for a 10-15 min appointment. If the outcomes of these "virtually managed" patients are similar to those seen physically, telemedicine can be adopted as a future standard-of-care. For the students, the virtual meetings offer an opportunity to participate and learn from conferences that are often unaffordable when conducted in distant places. Covid-related research and literature is now easily available through a number of online sites. An excellent resource is LitCovid at https://www.ncbi.nlm.nih. gov/research/coronavirus/ especially created to help in coronavirus research [5].

Therapeutic interventions to manage Covid-19 are evolving. Steroids were considered a contraindication for infected patients. The UK RECOVERY trial showed that a short course of dexamethasone reduced mortality in patients who require respiratory support, but had no benefit among those who did not require oxygen [6]. Some of the drugs on trial for Covid-19 are useful in various hematological disorders, such as corticosteroids, ruxolitinib, tocilizumab, anticoagulants, decitabine, imatinib, and cyclosporine, to mention a few. Registered interventional trials related to Covid-19 can be accessed online at www. covid-trials.org. Over time, the greater patient survival from Covid-19 suggests that therapy of severe disease has improved [7]. We need to know if this is also true for patients with hematological diseases.

Management of Covid-19 patients is highly variable, based on national policies, institutional recommendations or office practice. There is one spectrum of patients who receive a combination of drugs which may include: azithromycin, hydroxychloroquine, favipiravir, LMWH, ivermectin, dexamethasone, convalescent plasma, remdesivir- along with traditional medicines and "immune boosting" agents. These therapies are likely based on emerging data, need to "do something rather than nothing" and demand by patients based on "social-media medical experts". Another group of patients may get only supportive treatment. Collection of institutional data is important and creation of a national hematology registry would help in evaluating outcomes of Covid-19 in different hematological disorders while evaluating the benefits (or harms) of these interventions. While this would be retrospective data, the potentially large number of patients would provide "real-world" experience that may be hypothesis-generating.

The SARS-Cov-2 virus is not going to disappear soon. It may mutate and change in virulence, and eventually it will be contained through public health measures, vaccinations and therapeutic interventions. Scientists must ensure that solutions are evidence-based, and not approved prematurely due to political interference. The hematology community should ensure optimal care for their patients by adapting to the challenges posed by this virus.

\section{References}

1. https://covid19.who.int/. WHO Coronairus Disease (COVID-19) Dashboard

2. Mehta V, Goel S, Kabarriti R, Cole D, Goldfinger M, AcunaVillaorduna A et al (2020) Case fatality rate of cancer patients with COVID-19 in a New York hospital system. Cancer Discov 10(7):935-941

3. Jain A, Singh C, Dhawan R, Jindal N, Mohindra R, Lad D et al (2020) How to use a prioritised approach for treating hematological disorders during the COVID-19 pandemic in India? Indian J Hematol Blood Transfus. https://doi.org/10.1007/s12288-02001300-0

4. Aggarwal M, Dass J, Mahapatra M (2020) Hemostatic abnormalities in COVID-19: an update. Indian J Hematol Blood Transfus. https://doi.org/10.1007/s12288-020-01328-2

5. Chen Q, Allot A, Lu Z (2020) Keep up with the latest coronavirus research. Nature 579(7798):193

6. Horby P, Lim WS, Emberson JR, Mafham M, Bell JL, Linsell L et al (2020) Dexamethasone in hospitalized patients with Covid19-preliminary report. N Engl J Med. https://doi.org/10.1056/ NEJMoa2021436

7. The Lancet Infectious D (2020) Curing COVID-19. Lancet Infect Dis. https://doi.org/10.1016/S1473-3099(20)30706-4

Publisher's Note Springer Nature remains neutral with regard to jurisdictional claims in published maps and institutional affiliations. 